\title{
El control interno: Un estudio analítico de los procesos contables en el centro de aplicación Productos Unión de la Universidad Peruana Unión
}

\author{
Internal control: An analytical study of the accounting processes in \\ the application center Products Unión de la Universidad Peruana \\ Unión
}

\author{
Salomón Áxel Vásquez Campos ${ }^{1 *}$, Merlin Arturo Lazo Palacios ${ }^{1}$ \\ ${ }^{1}$ Escuela Profesional de Contabilidad, Facultad de Ciencias Empresariales, Universidad Peruana Unión
}

\section{INFORMACIÓN DEL ARTÍCULO}

Historia del artículo

Recibido: 24 de agosto 2016

Aceptado: 13 de octubre del 2016

\section{Palabras clave:}

Control interno, efectivo en caja y bancos, créditos y cobranzas, inventarios.

\begin{abstract}
Resumen
El objetivo del presente trabajo de investigación es analizar el estado del control interno en el centro de aplicación Productos Unión de la Universidad Peruana Unión, 2014. El tipo de estudio realizado es descriptivo con un diseño no experimental. La población está constituida por el total de personas involucradas en el área financiera del centro de aplicación. La recolección de datos se realizó a través de un cuestionario para el análisis de los procesos llevados a cabo en el área financiera. Según el análisis descriptivo, los trabajadores opinan que el control interno en general, el control interno del efectivo en caja y banco, el control interno en créditos y cobranzas y control interno de inventarios se encuentra en el nivel de excelente. Según el análisis de dependencia se tiene como resultados: 1) no hay dependencia entre las variables título profesional y control interno sobre el efectivo en caja y bancos. 2) no hay dependencia entre las variables título profesional y control interno en créditos y cobranzas, 3) no hay dependencia entre las variables título profesional y control interno sobre los inventarios, 4) no hay dependencia entre las variables grados de estudios y control interno sobre el efectivo en caja y bancos, 5) no hay dependencia entre las variables grados de estudios y control interno en créditos y cobranzas, 6) no hay dependencia entre las variables grados de estudios y control interno sobre los inventarios.
\end{abstract}

\section{Keywords:}

Internal control, cash holdings and banks, credit and collections, inventories.

\begin{abstract}
Abstrac
The aim of this research was to analyze the state of the internal control in the Implementation Center Productos Unión of the Peruvian Union University, 2014. A descriptive study was conducted with a non-experimental design. The study population consists of the total of people involved in the financial area of the Implementation Center Productos Unión. The collection of data was performed through a questionnaire for the analysis of the processes carried out in the financial area. According to the descriptive analysis the workers think that I hospitalize the control in general, the internal control of the cash in box and bank, the internal control in credits and collections
\end{abstract}


and internal control of inventories is in the level of excellently. According to the analysis of dependence it is had as results: 1) there is no dependence between the variables professional tittle and internal control over cash holdings and banks, 2) there is no dependence between the variables professional tittle and internal control over credit and collections, 3) there is no dependence between the variables professional tittle and internal control over inventories, 4) there is no dependence between the variables levels of educational and internal control over cash holdings and banks, 5) there is no dependence between the variables levels of educational and internal control over credit and collections, 6) there is no dependence between the variables levels of educational and internal control over inventories.

\section{Introducción}

Según Márquez, Visnellys, Rojas y Eucaris (2011), el control interno es una herramienta de suma importancia para salvaguardar y proteger aspectos de índole financieros, económicos, entre otros, debido a que su buena utilización reduce en gran magnitud las ilegalidades y corrupción dentro del marco laboral. Este proceso de control es llevado a cabo por la alta gerencia, funcionario y directores (Lam 2002).

La investigación de Asubadin (2011), que intenta estudiar las consecuencias del control interno del proceso de compras para determinar la incidencia en la liquidez de la empresa Comercial Yucailla, durante el año 2010, llega a determinar que control interno es importante puesto que ayuda a formar un buen ambiente de trabajo y, sobre todo, orienta al personal y gerentes de la institución a cumplir las funciones encomendadas con eficiencia y eficacia, logrando cumplir las metas planteadas. También Zobeida y Hiralid (2007) en su investigación evalúa el control interno del Departamento de Almacén del Centro Estatal de Coordinación Falcón, concluyendo que el cumplimiento de las políticas y mecanismos de control físico permite asegurar todos los activos físicos vulnerables, en caso de que no exista un seguro contra los riesgos a los cuales estos se exponen.
La importancia del control interno en la empresa es relevante ya que afectan las políticas, procedimientos, estructura de la organización, métodos para asignar autoridad y responsabilidad e, incluso el funcionamiento del consejo de la administración, así como su relación con la confiabilidad de los estados financieros, sistemas de información, eficacia operativa y la reducción de riesgo de fraudes (Flores 2006).

Para la posible solución de problemas encontrados en las diferentes entidades, surgen los diferentes tipos de controles el control interno fiscal, que para Hernández (2011) resulta del cálculo confiable de los tributos a declarar y pagar, el mismo que comprende políticas y procedimientos fiscales, establecidos por los encargados de la dirección de la entidad y por todo el personal fiscal de la misma, de modo que existe certeza sobre la determinación de los impuestos según la normatividad vigente.

También el control interno financiero que para Vílchez (2008), es el plan de organización y métodos, procedimientos y registros del control y salvaguarda de los activos, la exactitud, confiabilidad y oportunidad de la presentación de información financiera, principalmente de autorización y aprobación, segregación de funciones, para el cumplimiento de los objetivos trazados por la gerencia 
En relación a la solución de problemas, y de acuerdo a la presente investigación, el control interno contable, según Márquez, Visnellys, Rojas y Eucaris (2011) está constituido por el plan de organización, métodos, procedimientos y registros relacionados con la custodia de recursos, con la exactitud, confiabilidad y oportunidad en la presentación de información contable, incluye controles sobre los sistemas de autorización y aprobación, segregación de funciones entre las operaciones de registro e información contable. Este tipo de control interno brinda seguridad razonable para que las operaciones sean ejecutadas de conformidad con las normas autorizadas y aprobadas por la administración, es decir las operaciones llegan a clasificarse, para ordernarse sistemáticamente, a fin de que se registren adecuadamente, con el propósito de elaborar los estados financieros, según los principios de contabilidad aceptados.

La propuesta del siguiente trabajo consiste en analizar el estado del control interno en los procesos contables del área financiera del centro de aplicación Productos Unión de la Universidad Peruana Unión 2014.

\section{Revisión de la literatura}

\section{Control interno}

Las normas de control interno, por su parte, constituyen lineamientos, criterios, métodos y disposiciones, muy válidos para la aplicación y regulación del control interno en las principales áreas, en este sentido, todas las normas de control interno son fundamentadas, para establecer los criterios y las prácticas de aceptación general, también aquellos lineamientos y estándares de control (Reyna 2013).
Según Ramos (2012), el control interno contable queda constituido por el plan de organización, los métodos, procedimientos y registros relacionados con la custodia de los recursos, con la exactitud, confiabilidad y oportunidad en la presentación de información contable, incluye controles sobre los sistemas de autorización y aprobación, segregación de funciones entre las operaciones de registro e información contable. El control interno administrativo comprende el plan de organización, políticas, procedimientos y prácticas, orientados a administrar las operaciones en una empresa, además permite asegurar el cumplimiento y metas establecidas, quedan incluidas actividades de planeación, organización, dirección y control de las operaciones, el sistema para presentar informes, medir y monitorear el desarrollo de las actividades (Román y Escobar, 2010).

\section{Importancia}

La importancia del control interno sobreentiende la protección de los activos en contra del desperdicio, el fraude y el uso ineficiente; se tiene a la exactitud y confiabilidad de los registros contables; se evalúa el cumplimiento de las políticas de la compañía y se verifica la eficiencia de las operaciones, en pocas palabras, el control interno está formado por todas las medidas que se toman para suministrar a la administración la seguridad de que todo está funcionando como se ha planificado y bajo ciertas normas establecidas (Meigs y Meigs, 1994, citado por Velasco, 2015). Además, porque se constituye en una herramienta de suma importancia, para salvaguardar y proteger aspectos de índole financieros, económicos, entre otros; su debida utilización hace que en las empresas permanezca un debido orden acorde con lo planificado por la gerencia, reduciendo, en gran magnitud, las ilegalidades y corrupción dentro del mar- 
co laboral, porque obliga a cada elemento de la organización a ser controlado por la persona pertinente (Márquez, Visnellys, Rojas y Eucaris, 2011).

\section{Componentes del control interno}

Según los componentes, el control de ambiente exige la buena conducta y disciplina de las personas que trabajan en la institución, teniendo en cuenta la integridad y competencia personal de una organización (Amado, 2014); siendo los valores éticos, fundamentales por ascendencia en los demás componentes (Morales, 2012). Otros factores dentro de este componente se encuentran: la filosofía de la administración, la atención y guía proporcionados por el consejo de administración, el estilo operativo, la manera como la gerencia confiere autoridad y asigna responsabilidades, organiza y desarrolla a su personal (Parra, 2014).

La valoración de riesgos debe establecer mecanismos para identificar, analizar y valorizar los riesgos, con el propósito de manejar y minimizar las posibilidades de errores o irregularidades producidas que afecten la eficiencia, efectividad y economía de los recursos, un error se refiere a las actuaciones intencionales, generalmente fraudulentas (Nájera, De Paz, y Martínez, 2005).

Las actividades de control se llevan a cabo en todo el proceso de la organización, en todos los niveles y todas las funciones, estando dentro de éstos los procesos: aprobación, autorización, conciliaciones y otros, y pueden clasificarse en controles preventivos, detectivos, correctivos, manuales o de usuario, de cómputo o de tecnología de información y administrativos (Estupiñán, Gaitan, Milessi, Milessi, Moreno y Bailey, 2006).
El control de información y comunicación se realiza mediante sistemas de información que proveen la cantidad necesaria, precisión, oportunidad y confiabilidad y la misma debe ser necesaria para una administración eficaz y eficiente, identificándose la información y los mecanismos necesarios para recogerla oportunamente con el propósito de cumplir los objetivos (Araujo, 2010).

El control de supervisión tiene como objetivo asegurar el cumplimiento de los planes y que el proceso no sea interrumpido, tales actividades de monitoreo constante pueden ser implantadas en los propios procesos del negocio, a través de evaluaciones separadas de la operación; es decir, mediante auditoría interna o externa.

\section{Materiales y métodos}

El tipo de estudio de este trabajo de investigación es descriptivo porqué pretende analizar el estado del control interno en el centro de aplicación Productos Unión de la Universidad Peruana Unión. Tiene un diseño no experimental porque no se manipulará ninguna variable de estudio durante la investigación. Es transversal porque se tomarán los datos en un solo momento.

\section{Participantes}

Participaron en el estudio 12 trabajadores, personal de área financiera del Centro de Aplicación Productos Unión de la Universidad Peruana Unión, 2014. El criterio de inclusión responde a la remuneración por planilla y se excluyó del estudio personal que cumplía los seis meses de trabajo

\section{Instrumentos}

El instrumento que se utilizó es una adaptación realizada de tres cuestiona- 
rios elaborados por Márquez, Visnellys y Rojas C., Eucaris Del V. (2011), que evalúa el estado del control interno del efectivo en caja y bancos, aplicado en el área de créditos (12 ítemes); control interno de cuentas por cobrar, en el área de cobranzas (11 ítemes) y control interno de sobre inventarios, en almacen (12 ítemes)

\section{Resultados}

Los resultados preliminares consisten en el análisis descriptivo del estado del control interno y el análisis de dependencia con algunas variables demográficas.

\section{Análisis descriptivo}

Se presentan los resultados respecto a los niveles del control interno y de sus dimensiones medante el baremo: alto, medio y bajo.

Tabla 1

Nivel de control interno

\begin{tabular}{|c|c|c|c|c|c|}
\hline & & $\begin{array}{c}\text { Fre- } \\
\text { cuencia }\end{array}$ & $\begin{array}{l}\text { Porcen- } \\
\text { taje }\end{array}$ & $\begin{array}{l}\text { Porcenta- } \\
\text { je válido }\end{array}$ & $\begin{array}{l}\text { Porcentaje } \\
\text { acumulado }\end{array}$ \\
\hline & Bueno & 1 & 12,5 & 12,5 & 12,5 \\
\hline \multirow[t]{2}{*}{ Válidos } & Excelente & 7 & 87,5 & 87,5 & 100 \\
\hline & Total & 8 & 100 & 100 & \\
\hline
\end{tabular}

La Tabla 1 muestra que el 87,5\% de trabajadores del área financiera de Productos Unión, valoran el nivel de control interno en la categoría de "excelente"; el $12,5 \%$ valoran en la categoría de "bueno". Ningún trabajador evalúa en la categoría de "malo". Para el análisis se consideró la escala de valoración: 1) 35-58 puntos = malo; 2) 59-81 puntos=bueno y 3) 82-105 puntos=excelente.
Tabla 2

Nivel de control interno de efectivo en caja y bancos

\begin{tabular}{cccccc}
\hline & & $\begin{array}{c}\text { Fre- } \\
\text { cuencia }\end{array}$ & $\begin{array}{c}\text { Porcen- } \\
\text { taje }\end{array}$ & $\begin{array}{c}\text { Porcenta- } \\
\text { je válido }\end{array}$ & $\begin{array}{c}\text { Porcentaje } \\
\text { acumulado }\end{array}$ \\
\hline \multirow{2}{*}{ Válidos } & $\begin{array}{c}\text { Ex- } \\
\text { celente }\end{array}$ & 6 & 75 & 75 & 100 \\
& Total & 8 & 100 & 100 & \\
\hline
\end{tabular}

En la Tabla 2 se observa que el $75 \%$ de trabajadores del área financiera de Productos Unión, valoran el nivel de control interno del efectivo en caja y bancos en la categoría de "excelente"; el 25\% valoran en la categoría de "bueno". Ningún trabajador evalúa en la categoría de "malo". Para el análisis se consideró la siguiente escala de valoración: 1) 12-19 puntos=malo; 2) 20-27 puntos=bueno; y 3) 28-36 puntos $=$ excelente.

Tabla 3

Nivel de control interno en créditos y cobranzas

\begin{tabular}{cccccc}
\hline & & $\begin{array}{c}\text { Fre- } \\
\text { cuencia }\end{array}$ & $\begin{array}{c}\text { Porcen- } \\
\text { taje }\end{array}$ & $\begin{array}{c}\text { Porcenta- } \\
\text { je válido }\end{array}$ & $\begin{array}{c}\text { Porcentaje } \\
\text { acumulado }\end{array}$ \\
\hline \multirow{2}{*}{ Válidos } & $\begin{array}{c}\text { Ex- } \\
\text { celente }\end{array}$ & 7 & 16,5 & 16,5 & 16,5 \\
& Total & 8 & 100 & 100 & 100 \\
\hline
\end{tabular}

La Tabla 3 muestra que el 83,5\% de trabajadores del área financiera de Productos Unión, valoran el nivel de control interno en caja en la categoría de "excelente"; el 16,5\% valoran en la categoría de "bueno". Ningún trabajador evalúa en la categoría de "malo". Para el análisis se consideró la siguiente escala de valoración: 1) 11-18 puntos $=$ malo; 2) 29-25 puntos=bueno; y 3) 26-33 puntos=excelente. 
Tabla 4

Nivel de control interno en los inventarios

\begin{tabular}{cccccc}
\hline & & $\begin{array}{c}\text { Fre- } \\
\text { cuencia }\end{array}$ & $\begin{array}{c}\text { Porcen- } \\
\text { taje }\end{array}$ & $\begin{array}{c}\text { Porcenta- } \\
\text { je válido }\end{array}$ & $\begin{array}{c}\text { Porcentaje } \\
\text { acumulado }\end{array}$ \\
\hline \multirow{3}{*}{ Válidos } & $\begin{array}{c}\text { Ex- } \\
\text { celente }\end{array}$ & 6 & 71 & 71 & 100 \\
& Total & 8 & 100 & 100 & \\
\hline
\end{tabular}

En la Tabla 4 se observa que el $71 \%$ de trabajadores del área financiera de Productos Unión, valoran el nivel de control interno de los inventarios en la categoría de "excelente"; el 29\% valoran en la categoría de "bueno". Ningún trabajador evalúa en la categoría de "malo". Para el análisis se consideró la siguiente escala de valoración: 1) $12-19$ puntos=malo; 2) $20-27$ puntos $=-$ bueno; y 3) 28-36 puntos=excelente.

\section{Análisis de dependencia}

Prueba de hipótesis 1

- Hipótesis nula $\left(\mathrm{H}_{0}\right)$ : Las operaciones de control interno sobre el efectivo en caja y bancos no dependen del título del empleado del personal del área financiera del Centro de Aplicación Productos Unión.

- Hipótesis alterna $\left(\mathrm{H}_{1}\right)$ : Las operaciones de control interno sobre el efectivo en caja y bancos dependen del título del empleado del personal del área financiera del Centro de Aplicación Productos Unión.

Criterio de decisión

Si p 0.05, se acepta la hipótesis nula. $\left(\mathrm{H}_{0}\right)$ Si p 0.05, se rechaza la hipótesis nula

Tabla 5

Operaciones de control interno sobre el efectivo en caja y bancos y el título del empleado.

\begin{tabular}{|c|c|c|c|c|c|}
\hline & Valor & $g l$ & $\begin{array}{l}\text { Sig. asintótica } \\
\text { (bilateral) }\end{array}$ & $\begin{array}{c}\text { Sig. exacta } \\
\text { (bilateral) }\end{array}$ & $\begin{array}{l}\text { Sig. exacta } \\
\text { (unilateral) }\end{array}$ \\
\hline Chi-cuadrado de Pearson &, $381^{\mathrm{a}}$ & 1 & 0,537 & & \\
\hline Corrección por continuidad ${ }^{b}$ & 0 & 1 & 1 & & \\
\hline Razón de verosimilitudes & 0,622 & 1 & 0,43 & & \\
\hline Estadístico exacto de Fisher & & & & 1 & 0,75 \\
\hline Asociación lineal por lineal & 0,333 & 1 & 0,564 & & \\
\hline $\mathrm{N}$ de casos válidos & 8 & & & & \\
\hline
\end{tabular}

En la Tabla 5, se obtuvo que el valor de Chi-cuadrado es 0,381, con un p valor $=0,537$ y un nivel de significancia ( $\alpha=$ 0.005 o $\alpha=0.01$ ), permitiendo aceptar la hipótesis nula. Esto indica que las operaciones de control interno sobre efectivo en caja y bancos no dependen del título del empleado del área financiera del Centro de Aplicación Productos Unión.
Prueba de hipótesis 2

- Hipótesis nula $\left(\mathrm{H}_{0}\right)$ : Las operaciones de control interno en créditos y cobranza no dependen del título del empleado del personal del área financiera del Centro de Aplicación Productos Unión. 
- Hipótesis alterna $\left(\mathrm{H}_{1}\right)$ : Las operaciones de control interno en créditos y cobranza dependen del título del empleado del personal del área financiera del Centro de Aplicación Productos Unión.

\section{Criterio de decisión}

Si el p> 0.05, se acepta la hipótesis nula. $\left(\mathrm{H}_{0}\right)$

Si el p> 0.05, se rechaza la hipótesis nula $\left(H_{0}\right)$ y se acepta la hipótesis alterna $\left(H_{1}\right)$

Tabla 6

Operaciones de control interno en créditos y cobranzas y el título del empleado.

\begin{tabular}{|c|c|c|c|c|c|}
\hline & Valor & $g l$ & $\begin{array}{l}\text { Sig. asintótica } \\
\text { (bilateral) }\end{array}$ & $\begin{array}{l}\text { Sig. exacta } \\
\text { (bilateral) }\end{array}$ & $\begin{array}{l}\text { Sig. exacta } \\
\text { (unilateral) }\end{array}$ \\
\hline Chi-cuadrado de Pearson & $1,905^{\mathrm{a}}$ & 1 & .168 & & \\
\hline Corrección por continuidadb & .076 & 1 & .783 & & \\
\hline Razón de verosimilitudes & 2.209 & 1 & .137 & & \\
\hline Estadístico exacto de Fisher & & & & .375 & .375 \\
\hline Asociación lineal por lineal & 1.667 & 1 & .197 & & \\
\hline $\mathrm{N}$ de casos válidos & 8 & & & & \\
\hline
\end{tabular}

a. 4 casillas $(100,0 \%)$ tienen una frecuencia esperada inferior a 5 . La frecuencia mínima esperada es ,38.

b. Calculado sólo para una tabla de $2 \times 2$.

En la Tabla 6, se obtuvo el valor de Chi-cuadrado es 1,905, con un $p$ valor $=0,168$ y un nivel de significancia ( $\alpha=$ 0.005 o $\alpha=0.01$ ), permitiendo aceptar la hipótesis nula. Esto indica que las operaciones de control interno créditos y cobranzas no dependen del título del empleado del área financiera del Centro de Aplicación Productos Unión.

\section{Prueba de hipótesis 3}

- Hipótesis nula $\left(\mathrm{H}_{0}\right)$ : Las operaciones de control interno de inventarios no dependen del título del empleado del personal del área fi- nanciera del Centro de Aplicación Productos Unión.

- Hipótesis alterna $\left(\mathrm{H}_{1}\right)$ : Las operaciones de control interno de inventarios dependen del título del empleado del personal del área financiera del Centro de Aplicación Productos Unión.

\section{Criterio de decisión}

Si p 0.05, se acepta la hipótesis nula. $\left(\mathrm{H}_{0}\right)$ Si p 0.05, se rechaza la hipótesis nula $\left(\mathrm{H}_{0}\right)$ y se acepta la hipótesis alterna $\left(\mathrm{H}_{1}\right)$ 
Tabla 7

Operaciones de control interno sobre los inventarios y el título del empleado

\begin{tabular}{|c|c|c|c|c|c|}
\hline & Valor & gl & $\begin{array}{l}\text { Sig. asintótica } \\
\text { (bilateral) }\end{array}$ & $\begin{array}{l}\text { Sig. exacta } \\
\text { (bilateral) }\end{array}$ & $\begin{array}{l}\text { Sig. exacta } \\
\text { (unilateral) }\end{array}$ \\
\hline Chi-cuadrado de Pearson & $3,429^{a}$ & 1 & .064 & & \\
\hline Corrección por continuidad ${ }^{b}$ & .381 & 1 & .537 & & \\
\hline Razón de verosimilitudes & 3.256 & 1 & .071 & & \\
\hline Estadístico exacto de Fisher & & & & .250 & .250 \\
\hline Asociación lineal por lineal & 3.000 & 1 & .083 & & \\
\hline $\mathrm{N}$ de casos válidos & 8 & & & & \\
\hline
\end{tabular}

a. 3 casillas $(75,0 \%)$ tienen una frecuencia esperada inferior a 5. La frecuencia mínima esperada es ,25.

b. Calculado solo para una tabla de $2 \times 2$.

En la Tabla 7, se obtuvo el valor de Chi-cuadrado es 3,429, con un $p$ valor $=0,064$ y un nivel de significancia $(\alpha=$ $0.005 \circ \alpha=0.01$ ), permitiendo aceptar la hipótesis nula. Esto indica que las operaciones de control interno de inventarios no dependen del título del empleado del área financiera del Centro de Aplicación Productos Unión.

\section{Prueba de hipótesis 4}

- Hipótesis nula (H0): Las operaciones de control interno sobre el efectivo en caja y bancos no dependen del grado de estudios del personal del área financiera del
Centro de Aplicación Productos Unión.

- Hipótesis alterna (H1): Las operaciones de control interno sobre el efectivo en caja y bancos dependen del grado de estudios del personal del area financier del Centro de Aplicación Productos Unión.

\section{Criterio de decisión}

Si p 0.05, se acepta la hipótesis nula. $\left(H_{0}\right)$ Si p 0.05 , se rechaza la hipótesis nula

Tabla 8

Operaciones de control interno sobre el efectivo en caja y bancos y el grado de estudios

\begin{tabular}{|c|c|c|c|c|c|}
\hline & Valor & $\mathrm{gl}$ & $\begin{array}{l}\text { Sig. asintótica } \\
\text { (bilateral) }\end{array}$ & $\begin{array}{l}\text { Sig. exacta } \\
\text { (bilateral) }\end{array}$ & $\begin{array}{l}\text { Sig. exacta } \\
\text { (unilateral) }\end{array}$ \\
\hline Chi-cuadrado de Pearson &, $381^{\mathrm{a}}$ & 1 & .537 & & \\
\hline Corrección por continuidad ${ }^{b}$ & 0.000 & 1 & 1.000 & & \\
\hline Razón de verosimilitudes & .622 & 1 & .430 & & \\
\hline Estadístico exacto de Fisher & & & & 1.000 & .750 \\
\hline Asociación lineal por lineal & .333 & 1 & .564 & & \\
\hline $\mathrm{N}$ de casos válidos & 8 & & & & \\
\hline
\end{tabular}

a. 3 casillas $(75,0 \%)$ tienen una frecuencia esperada inferior a 5. La frecuencia mínima esperada es ,25.

b. Calculado solo para una tabla de $2 \times 2$. 
En la Tabla 8, se obtuvo que el valor de Chi-cuadrado es 0,381 , con un $p$ valor $=0,537$ y un nivel de significancia ( $\alpha=$ 0.005 o $\alpha=0.01$ ), permitiendo aceptar la hipótesis nula. Esto indica que las operaciones de control interno del efectivo en caja y bancos no dependen del grado de estudios del empleado del área financiera del Centro de Aplicación Productos Unión.

\section{Prueba de hipótesis 5}

- Hipótesis nula (H0): Las operaciones de control interno en créditos y cobranzas no dependen del gra- do de estudios del personal del área financiera del Centro de Aplicación Productos Unión.

- Hipótesis alterna (H1): Las operaciones de control interno en créditos y cobranzas dependen del grado de estudios del personal del área financiera del Centro de Aplicación Productos Unión.

\section{Criterio de decisión}

Si p 0.05, se acepta la hipótesis nula. $\left(\mathrm{H}_{0}\right)$ Si p 0.05, se rechaza la hipótesis nula

Tabla 9

Operaciones de control interno en créditos y cobranzas y el grado de estudios

\begin{tabular}{|c|c|c|c|c|c|}
\hline & Valor & $\mathrm{gl}$ & $\begin{array}{l}\text { Sig. asintótica } \\
\text { (bilateral) }\end{array}$ & $\begin{array}{l}\text { Sig. exacta } \\
\text { (bilateral) }\end{array}$ & $\begin{array}{l}\text { Sig. exacta } \\
\text { (unilateral) }\end{array}$ \\
\hline Chi-cuadrado de Pearson & $1,905^{\mathrm{a}}$ & 1 & .168 & & \\
\hline Corrección por continuidad ${ }^{\mathrm{b}}$ & .076 & 1 & .783 & & \\
\hline Razón de verosimilitudes & 2.209 & 1 & .137 & & \\
\hline Estadístico exacto de Fisher & & & & .375 & .375 \\
\hline Asociación lineal por lineal & 1.667 & 1 & .197 & & \\
\hline $\mathrm{N}$ de casos válidos & 8 & & & & \\
\hline
\end{tabular}

a. 4 casillas $(100,0 \%)$ tienen una frecuencia esperada inferior a 5 . La frecuencia mínima esperada es ,38.

b. Calculado solo para una tabla de $2 \times 2$.

En la Tabla 9, se obtuvo que el valor de Chi-cuadrado es 1,905, con un p valor $=0,168$ y un nivel de significancia $(\alpha$ $=0.005$ o $\alpha=0.01$ ), permitiendo aceptar la hipótesis nula. Esto indica que las operaciones de control interno créditos y cobranzas no dependen del grado de estudios del empleado del área financiera del Centro de Aplicación Productos Unión.

Prueba de hipótesis 6

- Hipótesis nula $\left(\mathrm{H}_{0}\right)$ : Las operaciones de control interno de inven- tarios no dependen del grado de estudios del personal del área financiera del Centro de Aplicación Productos Unión.

- Hipótesis alterna $\left(\mathrm{H}_{1}\right)$ : Las operaciones de control interno de inventarios dependen del grado de estudios del personal del área financiera del Centro de Aplicación Productos Unión. 


\section{Criterio de decisión}

Si p 0.05, se acepta la hipótesis nula. $\left(\mathrm{H}_{0}\right)$

Si p 0.05, se rechaza la hipótesis nula

Tabla 10

Operaciones de control interno sobre los inventarios y el grado de estudios

\begin{tabular}{|c|c|c|c|c|c|}
\hline & Valor & $g l$ & $\begin{array}{l}\text { Sig. asintótica } \\
\text { (bilateral) }\end{array}$ & $\begin{array}{l}\text { Sig. exacta } \\
\text { (bilateral) }\end{array}$ & $\begin{array}{l}\text { Sig. exacta } \\
\text { (unilateral) }\end{array}$ \\
\hline Chi-cuadrado de Pearson & $3,429^{a}$ & 1 & .064 & & \\
\hline Corrección por continuidad ${ }^{b}$ & .381 & 1 & .537 & & \\
\hline Razón de verosimilitudes & 3.256 & 1 & .071 & & \\
\hline Estadístico exacto de Fisher & & & & .250 & .250 \\
\hline Asociación lineal por lineal & 3.000 & 1 & .083 & & \\
\hline $\mathrm{N}$ de casos válidos & 8 & & & & \\
\hline
\end{tabular}

a. 3 casillas $(75,0 \%)$ tienen una frecuencia esperada inferior a 5. La frecuencia mínima esperada es ,25.

b. Calculado solo para una tabla de $2 \times 2$.

En la Tabla 10, se obtuvo que el valor de Chi-cuadrado es 3,429 , con un p valor $=0,064$ y un nivel de significancia ( $\alpha=$ 0.005 o $\alpha=0.01$ ), permitiendo aceptar la hipótesis nula. Esto indica que las operaciones de control interno de inventarios no dependen del grado de estudios del empleado del área financiera del Centro de Aplicación Productos Unión.

\section{Conclusiones}

\section{Conclusiones del nivel de control interno}

- El $75 \%$ de la percepción del total de trabajadores del área financiera de Productos Unión sobre el nivel de control interno en caja se ubica en la categoría de excelente y el $25 \%$ considera que el control interno es bueno.

- El 87,5\% de la percepción del total de trabajadores del área financiera de Productos Unión sobre el nivel de control interno en crédito y co- branzas se ubica en la categoría de excelente y el $12,5 \%$ considera que el control interno es bueno.

- El $71 \%$ de la percepción del total de trabajadores del área financiera de Productos Unión sobre el nivel de control interno en los inventarios se ubica en la categoría de excelente y el $29 \%$ considera que el control interno es bueno.

- El 83,5\% de la percepción del total de trabajadores del área financiera de Productos Unión sobre el nivel de control interno se ubica en la categoría de excelente y el $16,5 \%$ considera que el control interno es bueno.

Conclusiones del análisis de dependencia

- Con un valor de Chi-cuadrado de 0,381, con 1 grado de libertad y significancia de $p=0,537(\alpha$ $=0.005$ o $\alpha=0.01$ ), para la primera 
hipótesis de trabajo se acepta la hipótesis nula, entonces no hay dependencia entre las variables título profesional y control interno sobre el efectivo en caja y bancos, esto es, las operaciones de control interno sobre efectivo en caja y bancos no dependen del título del empleado del centro de aplicación Productos Unión.

- Con un valor de Chi-cuadrado de 1,905, con 1 grado de libertad y significancia de $p=0,168(\alpha=$ 0.005 o $\alpha=0.01$ ), para la segunda hipótesis de trabajo se acepta la hipótesis nula, entonces no hay dependencia entre las variables título profesional y control interno en créditos y cobranzas, esto es, las operaciones de control interno en créditos y cobranzas no dependen del título del empleado del centro de aplicación Productos Unión.

- Con un valor de Chi-cuadrado de 3,429 , con 1 grado de libertad y significancia de $\mathrm{p}=0,064(\alpha$ $=0.005$ o $\alpha=0.01$ ), para la tercera hipótesis de trabajo se acepta la hipótesis nula, entonces no hay dependencia entre las variables título profesional y control interno sobre los inventarios, esto es, las operaciones de control interno sobre los inventarios no dependen del título del empleado del centro de aplicación Productos Unión.

- Con un valor de Chi-cuadrado es 0,381 , con 1 grado de libertad y significancia de $p=0,537(\alpha=0.005$ o $\alpha=0.01)$, para la cuarta hipótesis de trabajo se acepta la hipótesis nula, entonces no hay dependencia entre las variables grados de estudios y control interno sobre el efectivo en caja y bancos, esto es, las operaciones de control interno sobre efectivo en caja y bancos no dependen del grado de estudios del empleado del centro de aplicación Productos Unión.

- Con un valor de Chi-cuadrado es 1,905, con 1 grado de libertad y significancia de $p=0,168(\alpha$ $=0.005$ o $\alpha=0.01$ ), para la quinta hipótesis de trabajo se acepta la hipótesis nula, entonces no hay dependencia entre las variables grados de estudios y control interno en créditos y cobranzas, esto es, las operaciones de control interno en créditos y cobranzas no dependen del grado de estudios del empleado del centro de aplicación Productos Unión.

- Con un valor de Chi-cuadrado es 3,429 , con 1 grado de libertad y significancia de $p=0,064(\alpha=0.005$ o $\alpha=0.01)$, para la sexta hipótesis de trabajo se acepta la hipótesis nula, entonces no hay dependencia entre las variables grados de estudios y control interno sobre los inventarios, esto es, las operaciones de control interno sobre los inventarios no dependen del grado de estudios del empleado del centro de aplicación Productos Unión. 


\section{Referencias}

Amado, N. (2014). Responsabilidad social empresarial y el control interno. Caso de la PyME del rubro alimentación: Familia Grion SRL. Tesis de licenciatura. Universidad Nacional de Córdoba.

Araujo, B. P., \& Morales, L. E. (2010). Utilidad del sistema de información contable computarizado en la toma de decisiones de la Alcaldía del Municipio Escuque, Estado Trujillo. Tesis de licenciatura. Universidad de los Andes.

Asubadin Azubadin, I. C. (2011). Control interno del proceso de compras y su incidencia en la liquidez de la empresa comercial Yucailla CIA. LTDA. Latacunga. Tesis para la obtención del título de Ingeniería en Contabilidad y Auditoría CPA. Universidad Técnica de Ambato, Facultad de Contabilidad y Auditoría, Carrera de Contabilidad y Auditoría. Ambato, Ecuador.

Estupiñán Gaitán, R., Gaitán, R. E., Holmes, A. W. A. W., Milessi, J. J., Milessi, C. E. J., Moreno, C. E., y Bailey, L. P. H. (2006). Control interno y fraudes con base en los ciclos transaccionales. Análisis de informe COSO I y II (No. 658.012. 7 658.4013). e-libro.

Flores Calderón, Y. (2006). Diseño de control interno para la pequeña y mediana empresa. Tesis para obtener el título de Licenciado en Contaduría. Universidad Autónoma del estado de Hidalgo. Facultad de Ciencias Económicas Administrativas. Pachuca.
Freire Villacrés, M. S. (2011). Relación de la evaluación del control interno con la rentabilidad en la empresa Merizalde e Hijos Cia. Ltda., en el año 2010. Tesis de licenciatura. Universidad Técnica de Ambato.

Hernández Campos, A. (2010). Análisis crítico del control interno fiscal y su incidencia en las contingencias fiscales en la industria manufacturera de Nuevo León. 2011. Tesis doctoral, Universidad Autónoma de Nuevo León, Facultad de Contaduría Pública y Administración, División de Estudios de Posgrado.

Lam Álvarez, Elsa María (2002). Evaluación del control interno basado en modelo coso. Tesis, Universidad Francisco Marroquín, Guatemala 2002.

Márquez, V., Visnellys, J., Rojas, C., y Eucaris, Del V (2011). Análisis del control interno del efectivo, cuentas por cobrar e inventario en la Empresa Fremendz, C. A., ubicada en Cumaná, Estado Sucre. 2011. Trabajo de grado presentado como requisito parcial para optar al título de Licenciado en Contaduría Pública. Universidad de Oriente Núcleo de Sucre, Escuela de Administración, Departamento de Contaduría.

Morales Andrade, M. L. (2012). Propuesta del diseño e implementación de un sistema de control interno administativo, aplicado en la Empresa" Globalexs SA" dedicada a brindar servicios de courier. Tesis de licenciatura. Universidad Central de Ecuador.

Nájera Mejía, L. P., De Paz Gavidia, J. N., \& Martínez Barahona, I. I. (2005). 
Auditoría financiera y fiscal integrada, como herramienta para optimizar recursos y minimizar costos. Tesis de licenciatura. Universidad de El Salvador.

Parra, L. I. C. (2014). Los sistemas de control interno en las Mipymes y su impacto en la efectividad empresarial. Revista En-Contexto, 2(2).

Ramos Morales, C. J. (2012). Efectos del control interno en las áreas administrativas del hospital nivel II Babahoyo de la Provincia de Los Ríos del Instituto Ecuatoriano de Seguridad Social. Tesis de grado. Universidad Técnica de Babahoyo.

Reyna Blas, M. E. (2013). Propuesta de implementación de un sistema de control interno y su incidencia en la gestión de la empresa constructora A \& V Inversiones SAC del distrito de Trujillo período 2011. Tesis de licenciatura de Contador Público. Universidad Nacional de Trujillo.

Román Pereira, N. L., y Escobar Torres, M. P. (2010). La ética y práctica profesional del personal en el cumplimiento de las normas de control interno en la información financiera de la Farmacia Nacional de la Ciu- dad de Loja en el periodo 2009. Tesis de grado. Universidad Nacional de Loja.

Velasco Herrera, V. D. C. (2015). El control interno y el cumplimiento de objetivos operacionales en la empresa industrial textiles Jhonatex en la ciudad de Ambato. Tesis de licenciatura. Universidad Técnica de Ambato.

Vílchez Cisneros, D. (2008). Control interno y su incidencia en la ejecución de gastos en la municipalidad provincial de Huamanga periodos: 2006-2007. Trabajo para optar el título profesional de Contador Público. Universidad Nacional de San Cristóbal de Huamanga. Contabilidad y Auditoría.

Zobeida Jimenez, H. (2007). Evaluación del control interno del departamento de almacén del centro estadal de coordinación falcón del ministerio del poder popular para la infraestructura. Trabajo para optar al grado de Especialista en Auditoría. Universidad Centro Occidental Lizandro Alvarado, convenio UCLA - LUZ, Postgrado: Especialización en contaduría, Mención: Auditoría. 
